\title{
Hierarchical Clustering Using Mutual Information
}

\author{
Alexander Kraskov, Harald Stögbauer, Ralph G. Andrzejak, and Peter Grassberger \\ John-von-Neumann Institute for Computing, Forschungszentrum Jülich, D-52425 Jülich, Germany
}

(Dated: October 23, 2018)

\begin{abstract}
We present a method for hierarchical clustering of data called mutual information clustering (MIC) algorithm. It uses mutual information (MI) as a similarity measure and exploits its grouping property: The MI between three objects $X, Y$, and $Z$ is equal to the sum of the MI between $X$ and $Y$, plus the MI between $Z$ and the combined object $(X Y)$. We use this both in the Shannon (probabilistic) version of information theory and in the Kolmogorov (algorithmic) version. We apply our method to the construction of phylogenetic trees from mitochondrial DNA sequences and to the output of independent components analysis (ICA) as illustrated with the ECG of a pregnant woman.
\end{abstract}

Classification or organizing of data is very important in all scientific disciplines and is fundamental for understanding and learning [1]. Classification can be exclusive or overlapping, supervised or unsupervised. In the following we will be interested only in exclusive unsupervised classification, called clustering.

An instance of a clustering problem consist of a set of objects and a set of properties (called characteristic vector) for each object. The goal of clustering is separation of objects into groups using only the characteristic vectors. Cluster analysis organizes data either as a single grouping of individuals into non-overlapping clusters or as a hierarchy of nested partitions. The latter is called hierarchical clustering (HC). Because of wide spread of applications, there are a large variety of different clustering methods in usage, see e.g. 1] for an overview.

The crucial point of all clustering algorithms is the choice of a proximity measure. This is obtained from the characteristic vectors and can be either an indicator for similarity or dissimilarity. In the latter case it is convenient but not obligatory to satisfy the standard axioms of a metric (positivity, symmetry, and triangle inequality). Among $\mathrm{HC}$ methods one should distinguish between those where one uses the characteristic vectors only at the first level of the hierarchy and derives the proximities between clusters from the proximities of their constituents, and methods where the proximities are calculated each time from their characteristic vectors. The latter strategy (which is used also in the present paper) allows of course for more flexibility but might also be computationally more costly.

Quite generally, the "objects" to be clustered can be either single (finite) patterns (e.g. DNA sequences) or random variables, i.e. probability distributions. In the latter case the data are usually supplied in form of a statistical sample, and one of the simplest and most widely used similarity measures is the linear (Pearson) correlation coefficient. But this is not sensitive to nonlinear dependencies which do not manifest themselves in the covariance and can thus miss important features. This is in contrast to mutual information (MI) which is also singled out by its information theoretic background [2]. Indeed, MI is zero only if the two random variables are strictly independent.

Another important feature of MI is that it has also an "algorithmic" cousin, defined within algorithmic (Kolmogorov) information theory [3] which measures the similarity between individual objects. For a thorough discussion of distance measures based on algorithmic MI and for their application to clustering, see [4, 5].

Essential for the present application is the grouping property of $\mathrm{MI}$,

$$
I(X, Y, Z)=I(X, Y)+I((X, Y), Z) .
$$

Within Shannon information theory this is an exact theorem, while it is true in the algorithmic version up to the usual logarithmic correction terms [3]. Since $X, Y$, and $Z$ can be themselves composite, Eq. (1) can be used recursively for a cluster decomposition of MI. This motivates the main idea of our clustering method: instead of using e.g. centers of masses in order to treat clusters like individual objects in an approximative way only, we treat them exactly like individual objects when using MI as proximity measure and We thus propose the following scheme for clustering $n$ objects with MIC:

(1) Compute a proximity matrix based on pairwise mutual informations; assign $n$ clusters such that each cluster contains exactly one object;

(2) find the two closest clusters $i$ and $j$;

(3) create a new cluster $(i j)$ by combining $i$ and $j$;

(4) delete the lines/columns with indices $i$ and $j$ from the proximity matrix, and add one line/column containing the proximities between cluster $(i j)$ and all other clusters;

(5) if the number of clusters is still $>2$, goto (2); else join the two clusters and stop.

Shannon Theory: Here, $X \equiv X_{1}, Y \equiv X_{2}, \ldots$ are random variables. If they are discrete, entropies are defined as usual $H(X)=-\sum_{i} p_{i}(X) \log p_{i}(X)$ etc. The MI is defined as

$$
I\left(X_{1}, \ldots, X_{n}\right)=\sum_{k=1}^{n} H\left(X_{k}\right)-H\left(X_{1}, \ldots, X_{n}\right) .
$$

Eq.(1) can be checked easily, together with its generalization to arbitrary groupings. It means that MI can be 
decomposed into hierarchical levels. By iterating it, one can decompose $I\left(X_{1} \ldots X_{n}\right)$ for any $n>2$ and for any partitioning of the set $\left(X_{1} \ldots X_{n}\right)$ into the MIs between elements within one cluster and MIs between clusters.

For continuous variables with densities $\mu_{X}$ etc., one first introduces some binning ('coarse-graining'), and applies the above to the binned variables. If $x$ is a vector with dimension $m$ and each bin has Lebesgue measure $\Delta$, then $p_{i}(X) \approx \mu_{X}(x) \Delta^{m}$ with $x$ chosen suitably in bin $i$, and

$$
H_{\mathrm{bin}}(X) \approx \tilde{H}(X)-m \log \Delta
$$

where the differential entropy is given by

$$
\tilde{H}(X)=-\int d x \mu_{X}(x) \log \mu_{X}(x) .
$$

Notice that $H_{\text {bin }}(X)$ is a true (average) information and is thus non-negative, but $\tilde{H}(X)$ is not an information, can be negative, and is not invariant under homeomorphisms $x \rightarrow \phi(x)$.

Joint entropies, conditional entropies, and MI are defined as above, with sums replaced by integrals. Like $\tilde{H}(X)$, joint and conditional entropies are neither positive (semi-)definite nor invariant. But MI, defined as

$$
I(X, Y)=\iint d x d y \mu_{X Y}(x, y) \log \frac{\mu_{X Y}(x, y)}{\mu_{X}(x) \mu_{Y}(y)},
$$

is non-negative and invariant under $x \rightarrow \phi(x)$ and $y \rightarrow$ $\psi(y)$. It is (the limit of) a true information,

$$
I(X, Y)=\lim _{\Delta \rightarrow 0}\left[H_{\mathrm{bin}}(X)+H_{\mathrm{bin}}(Y)-H_{\mathrm{bin}}(X, Y)\right] .
$$

In applications, one usually has the data available in form of $N$ sample points $\left(x_{i}, y_{i}\right), i=1, \ldots N$ which are assumed to be i.i.d. realizations. There exist numerous algorithms to estimate $I(X, Y)$ and entropies. We use in the following the MI estimators proposed recently in Ref. [6], and we refer to this paper for a review of alternative methods.

Algorithmic Information Theory: In contrast to Shannon theory where the basic objects are random variables and entropies are average informations, algorithmic information theory deals with individual symbol strings and with the actual information needed to specify them. To "specify" a sequence $X$ means here to give the necessary input to a universal computer $U$, such that $U$ prints $X$ on its output and stops. The analogon to entropy, called here usually the complexity $K(X)$ of $X$, is the minimal length of an input which leads to the output $X$, for fixed $U$. It depends on $U$, but it can be shown that this dependence is weak and can be neglected in the limit when $K(X)$ is large [3].

Let us denote the concatenation of two strings $X$ and $Y$ as $X Y$. Its complexity is $K(X Y)$. It is intuitively clear that $K(X Y)$ should be larger than $K(X)$ but cannot be larger than the sum $K(X)+K(Y)$. Finally, one expects that $K(X \mid Y)$, defined as the minimal length of a program printing $X$ when $Y$ is furnished as auxiliary input, is related to $K(X Y)-K(Y)$. Indeed, one can show [3] (again within correction terms which become irrelevant asymptotically) that

$$
0 \leq K(X \mid Y) \simeq K(X Y)-K(Y) \leq K(X) .
$$

Notice the close similarity with Shannon entropy. The algorithmic information in $Y$ about $X$ is finally

$I_{\text {alg }}(X, Y)=K(X)-K(X \mid Y) \simeq K(X)+K(Y)-K(X Y)$,

and similarly for more than two strings. Within the same additive correction terms, one shows that it is symmetric, $I_{\text {alg }}(X, Y)=I_{\text {alg }}(Y, X)$, and can thus serve as an analogon to mutual information.

$K(X)$ is in general not computable. But one can easily give upper bounds: The length of any input which produces $X$ (e.g. by spelling it out verbatim) is an upper bound. Improved upper bounds are provided by any file compression algorithm.

MI-Based Distance Measures: When comparing objects with different marginal or joint informations, it seems intuitively clear that one should prefer relative distances over absolute ones, in order to minimize the dependence on the total information. We here use the quantity [4, 7]

$$
D(X, Y)=1-\frac{I(X, Y)}{H(X, Y)}
$$

which is a metric, with $D(X, X)=0$ and $D(X, Y) \leq$ 1 for all pairs $(X, Y)$. The algorithmic version is also universal: If $X \approx Y$ according to any non-trivial distance measure, then $X \approx Y$ also according to $D$.

A difficulty appears in the Shannon framework, if we deal with continuous random variables. As we mentioned above, $\tilde{H}(X, Y)$ is not invariant under homeomorphisms (including rescalings) and not even positive definite, while $H_{\text {bin }}$ diverges when $\Delta \rightarrow 0$. We thus modified Eq.(9) by replacing $H(X, Y)$ by $H_{\text {bin }}(X, Y)$ and replacing $D(X, Y)$ by the similarity measure

$$
S(X, Y)=\lim _{\Delta \rightarrow 0}(D(X, Y)-1) \log \Delta=\frac{I(X, Y)}{m_{x}+m_{y}} .
$$

A Phylogenetic Tree for Mammals: We study the mitochondrial DNA of a group of 34 mammals (see Fig. 1). The same data [8] had previously been analyzed in [4, 9]. This group includes among others some rodents, ferungulates, and primates.

Obviously we are here dealing with the algorithmic version of information theory, and informations are estimated by lossless data compression. For constructing the proximity matrix between individual taxa, we proceed essentially as in Ref. [4], using the special compression program GenCompress [10]. 


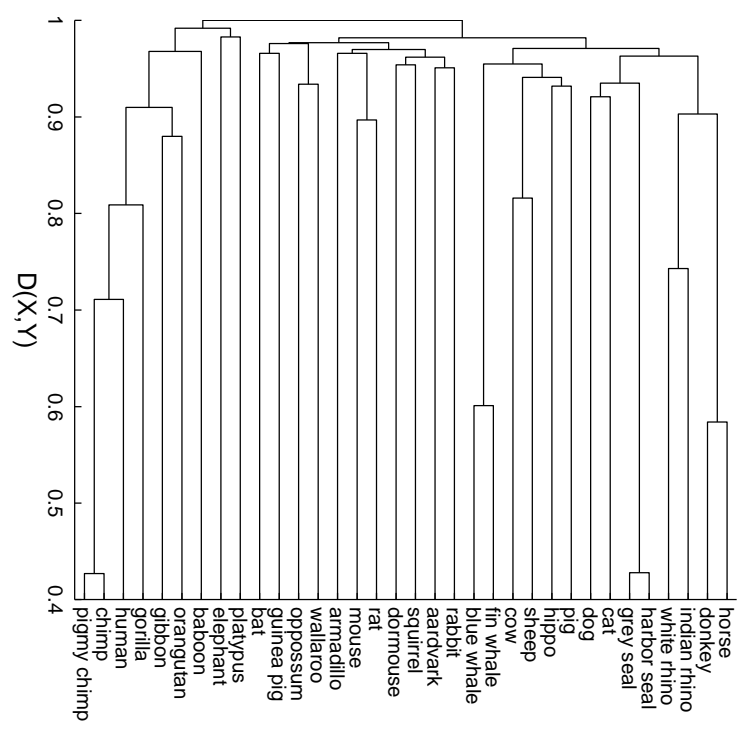

FIG. 1: Phylogenetic tree for 34 mammals. The heights of nodes are the distances between the joining daughter clusters.

In Ref. [4], this proximity matrix was then used as the input to a standard $\mathrm{HC}$ algorithm (neighbour-joining and hypercleaning) to produce an evolutionary tree. Instead we use the MIC algorithm with distance $D(X, Y)$. The joining of two clusters is obtained by simply concatenating the DNA sequences. There is of course an arbitrariness in the order of concatenation sequences: $X Y$ and $Y X$ give in general compressed sequences of different lengths. But we found this to have negligible effect on the evolutionary tree.

The overall structure of this tree closely resembles the one shown in Ref. [9]. All primates are correctly clustered and also the relative order of the ferungulates (blue whale to horse) is in accordance with Ref. [9]. On the other hand, there are a number of connections which obviously do not reflect the true evolutionary tree, see for example the guinea pig with bat and elephant with platypus. But the latter two, inspite of being joined together, have a very large distance from each other, thus their clustering just reflects the fact that neither the platypus nor the elephant have other close relatives in the sample. All in all, however, already the results shown in Fig. 1 capture surprisingly well the overall structure shown in Ref. [9]. Dividing MI by the total information is essential for this success. If we had used the non-normalized $I_{\text {alg }}(X, Y)$ itself, the clustering algorithm used in [4] would not change much, since all 34 DNA sequences have roughly the same length. But our MIC algorithm would be completely screwed up: After the first cluster formation, we have DNA sequences of very different lengths, and longer sequences tend also to have larger MI, even if they are not closely related.

The concatenation of $X$ and $Y$ will of course not lead to a plausible sequence of the common ancestor, but it optimally represents the information about it. This information is essential to find the correct way through higher hierarchy levels of the evolutionary tree, and it is preserved in concatenating.

Clustering of Minimally Dependent Components in an Electrocardiogram: As our second application we choose a case where Shannon theory is the proper setting. We show in Fig. 2 an ECG recorded from the abdomen and thorax of a pregnant woman [11]. It is already seen from this graph that there are at least two important components in this ECG: the heartbeat of the mother and of the fetus. In addition there is noise from various sources (muscle activity, measurement noise, etc.). While it is easy to detect anomalies in the mother's ECG from such a recording, it would be difficult to detect them in the fetal ECG.

As a first approximation we can assume that the total ECG is a linear superposition of several independent sources (mother, child, noise 1, noise $_{2}, \ldots$ ). A standard method to disentangle such superpositions is independent component analysis (ICA) [12]. There, one tries to recover the sources by means of linear transformation $s_{i}(t)=\sum_{j=1}^{n} W_{i j} x_{j}(t)$, where $W_{i j}$ is determined by minimizing the estimated MI between the $s_{i}$.

In reality things are not so simple. For instance, the sources might not be independent, the number of sources (including noise sources!) might be different from the number of channels, and the mixing might involve delays. For the present case this implies that the heartbeat of the mother is seen in several reconstructed components $s_{i}$, and that the "independent" components are not independent at all. In particular, all components $s_{i}$ which have large contributions from the mother form a cluster with large intra-cluster MIs and small inter-cluster MIs. The same is true for the fetal ECG, albeit less pronounced. To obtain clean recordings of the fetal and maternal ECGs, we proceeded as follows [13.

Since we expect different delays in the different channels, we first used Takens delay embedding [14] with time delay $0.002 \mathrm{~s}$ and embedding dimension 3 , resulting in 24 channels. We then formed 24 linear combinations $s_{i}(t)$. We use the MI estimator [ $[$ ], for details see [7]. Five of the resulting least dependent components contain strong contributions of the mother's heartbeat, three are dominated by the fetus. The rest contains mostly noise [7].

In plotting the actual dendrogram (Fig. 3) we used $S(X, Y)$ for the cluster analysis but used the MI of the clusters to determine the height at which the two branches join. The MI of the first five channels, e.g., is $\approx 1.44$ nats, while that of channels 6 to 8 is $\approx 0.3$ nats. For any two clusters (tuples) $X=X_{1} \ldots X_{n}$ and $Y=$ $Y_{1} \ldots Y_{m}$ one has $I(X, Y) \geq I(X)+I(Y)$. This guarantees, if the MI is estimated correctly, that the tree is drawn properly. The two slight glitches (when clusters $(1-14)$ and $(15-18)$ join, and when $(21-22)$ is joined with 23) result from small errors in estimating MI. They 


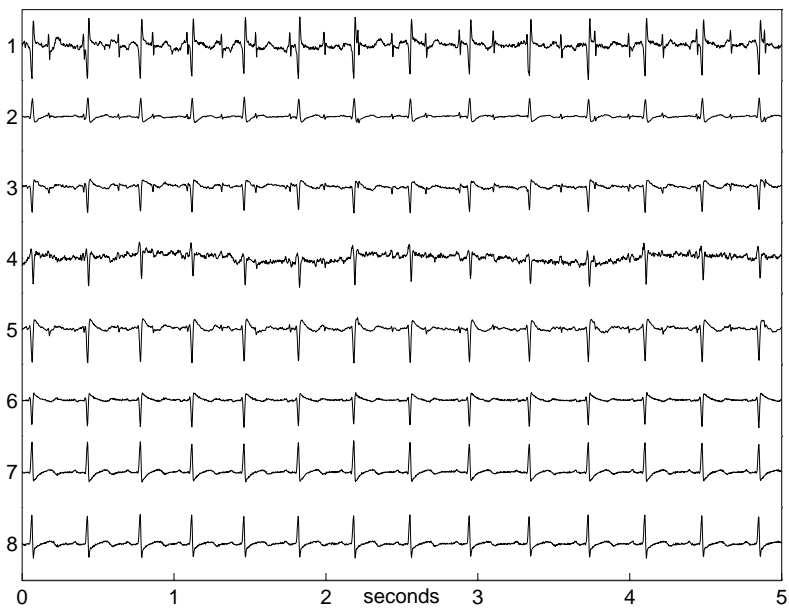

FIG. 2: ECG of a pregnant woman (sampling rate $500 \mathrm{~Hz}$ ).

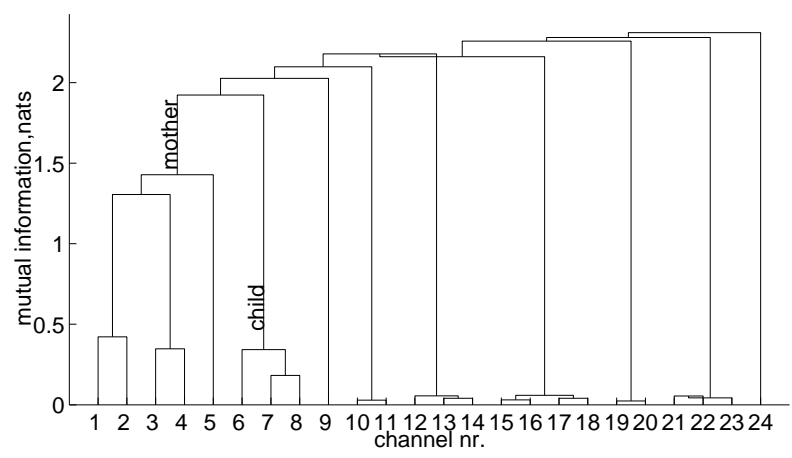

FIG. 3: Dendrogram for least dependent components.

do in no way effect our conclusions.

In Fig. 3] one can clearly see two big clusters corresponding to the mother and to the child. There are also some small clusters which should be considered as noise. For reconstructing the mother and child contributions to Fig. 2] we have to decide on one specific clustering from the entire hierarchy. We decided to make the cut at intercluster MI equal to 0.1, i.e. two clusters $X$ and $Y$ are joined whenever $I((X),(Y)) \equiv I(X, Y)-I(X)-I(Y) \geq$ 0.1. Reconstructing the first five traces of the original ECG from the child components only, we obtain Fig. [4

In summary, we have shown that MI can not only be used as a proximity measure in clustering, but that it also suggests a conceptually very simple and natural hierarchical clustering algorithm. We do not claim that this algorithm, called mutual information clustering (MIC), is always superior to other algorithms. Indeed, MI is in general not easy to estimate. Obviously, when only crude estimates are possible, also MIC will not give very good results. But as MI estimates are becoming better, also the results of MIC should improve. The present paper was partly triggered by the development of a new class of MI estimators for continuous random variables which have very small bias and also rather small variances [ $[$ ]

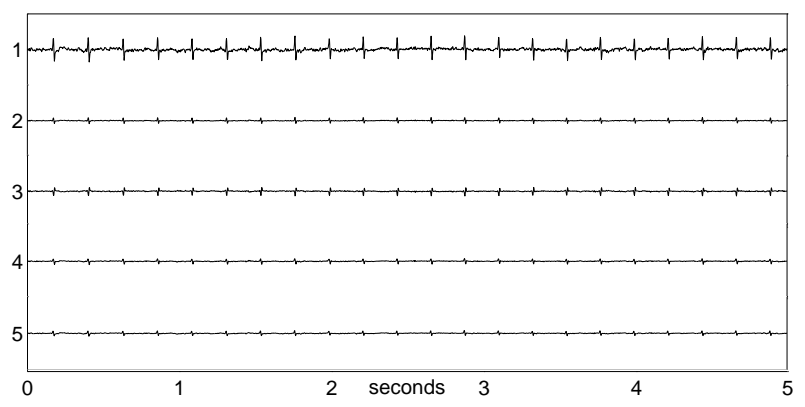

FIG. 4: ECG where all contributions except those of the child cluster have been removed.

We have illustrated our method with two applications, one from genetics and one from cardiology. For neither application MIC might give optimal clustering, but it seems promising that one common method gives decent results for both, although they are very different.

The results of MIC should improve, if more data become available. This is trivial, if we mean by that longer time sequences in the application to ECG, and longer parts of the genome. It is less trivial that we expect MIC to make fewer mistakes in a phylogenetic tree, when more species are included. The reason is that close-by species will be correctly joined anyhow, and families - which now are represented only by single species and thus are poorly characterized - will be much better described by the concatenated genomes if more species are included.

We would like to thank Arndt von Haesseler, Walter Nadler and Volker Roth for many useful discussions.

[1] A.K. Jain and R.C.Dubes, Algorithms for Clustering Data (Prentice Hall, Englewood Cliffs, NJ, 1988).

[2] T.M. Cover and J.A. Thomas, Elements of Information Theory (Wiley, New York 1991).

[3] M. Li and P. Vitanyi, An Introduction to Kolmogorov Complexity and its Applications, 2nd ed. (Springer, New York 1997).

[4] M. Li et al., Bioinformatics, 17, 149 (2001).

[5] M. Li et al., e-print CC/0111054 (2002).

[6] A. Kraskov, H. Stögbauer and P. Grassberger, e-print cond-mat/0305641 (2003).

[7] A. Kraskov, H. Stögbauer, R.G. Andrzejak, and P. Grassberger, to be published

[8] http://www.ncbi.nlm.nih.gov/

[9] A. Reyes et al., Mol. Biol. Evol. 17, 979 (2000).

[10] http://www.cs.ucsb.edu/ mli/Bioinf/software/index.html

[11] B.L.R. De Moor, ed., www.esat.kuleuven.ac.be/sista/daisy (1997).

[12] A. Hyvärinen, J. Karhunen, and E. Oja, Independent Component Analysis (Wiley, New York 2001).

[13] H. Stögbauer, A. Kraskov, and P. Grassberger, to be published

[14] F. Takens. In Dynamical Systems and Turbulence, eds. D.A. Rand and L.S. Young, Springer Lecture Notes in Mathematics 898, page 366 (Springer, Berlin 1980). 